\title{
Can we reduce the burden of the current UK guidelines for retinopathy of prematurity screening?
}

Eye (2018) 32, 235-237; doi:10.1038/eye.2017.163; published online 11 August 2017

Infants born more than 8-10 weeks preterm are at risk of developing sight-threatening retinopathy of prematurity (ROP). In the United Kingdom and other countries, paediatric ophthalmologists systematically screen infants at risk, with the aim of identifying ROP requiring treatment to prevent adverse structural outcomes, such as retinal detachment and macular dragging, and poor functional outcomes such as sight impairment.

ROP screening involves instillation of mydriatics, application of a lid speculum, and fundoscopy via indirect ophthalmoscopy or digital imaging, and is distressing for infants. Changes in blood pressure, respiratory rate, oxygen saturation and pulse rate, and facial changes indicative of pain are common. ${ }^{1-3}$ Repeated screening is required at weekly or twoweekly intervals either until ROP has spontaneously regressed, or a need for treatment has been established. ROP screening requires a skilled workforce available 52 weeks a year. Failure to identify infants requiring treatment at the appropriate time, as well as resulting in blindness for the premature infant, can have significant adverse medicolegal considerations. ${ }^{4}$ Over recent years, the increasing number of infants surviving preterm birth has resulted in an increased need for trained paediatric ophthalmologists. There is no universal consensus on the cutoff for gestational age (GA) that should determine the need for screening, and as ROP is a developmental disorder, it is illogical for birth weight (BW) to be included in the selection algorithm. The inclusion of BW likely arose before universal assignment of GA through early ultrasound assessment, and remains a historical anachronism. In the United
States, screening is recommended for GA of 30 weeks or less and BW of $1500 \mathrm{~g}$ or less (plus selected infants with a higher GA and BW and an unstable clinical course). ${ }^{5}$ In Canada, infants are screened if GA is $30+6 / 7$ or less, regardless of BW, or if BW is $1250 \mathrm{~g}$ or less. ${ }^{6}$ In Sweden, screening is undertaken for GA of 31 weeks or less, with no consideration of BW. ${ }^{7}$

The current UK guidelines (2008) recommend screening for infants with a GA of less than 32 weeks or BW less than 1501 g. ${ }^{8}$ We recently reported that of 8112 infants with BW less than $1500 \mathrm{~g}$ born over a one-year period in the United Kingdom and Northern Ireland, 327 (4\%) required ROP treatment. ${ }^{9}$ A revision of the UK ROP screening guidelines is now under consideration.

Is it possible to reduce the UK screening burden?

In our recent national study, the median GA of infants requiring ROP treatment was 25 weeks and the median BW 706 g. ${ }^{9}$ No baby was over 32 weeks GA and all were 31 weeks GA or less; only one baby had a BW over $1500 \mathrm{~g}$ (BW $2080 \mathrm{~g}$, GA 30+1 weeks, diabetic mother).

Tightening the UK screening criteria to reduce the number of infants screened unnecessarily should ensure that no cases of ROP requiring treatment are missed. Possible scenarios are to (1) keep the current GA indication of $31+6$ weeks while lowering the BW cutoff to less than $1251 \mathrm{~g}$, (2) lower the GA cutoff to $30+6$ weeks while keeping a BW of less than $1501 \mathrm{~g}$, or (3) lower both GA and BW cutoff (GA of 30+6 and birth weight of less than $1251 \mathrm{~g}$ ), (4) use GA only of 31 +6 or less, (5) use GA only of $30+6$ or less.

With information provided by the Neonatal Data Analysis Unit (NDAU) from the National Neonatal Research Database, we examined the effect any changes in screening criteria would have on the number of babies undergoing 
Table 1 Data on infants recorded in the National Neonatal Research Database (birth dates 1 December 2013-30 November 2014) and potential reduction in infants screened for ROP if UK screening guidelines tightened

\begin{tabular}{|c|c|c|c|c|c|c|c|c|}
\hline & \multirow[t]{2}{*}{ England } & \multirow[t]{2}{*}{ Scotland } & \multirow[t]{2}{*}{ Wales } & \multirow[t]{2}{*}{ Total } & \multicolumn{4}{|c|}{ Potential reduction in infants screened (\%) } \\
\hline & & & & & England & Scotland & Wales & Total \\
\hline \multicolumn{9}{|c|}{ Number of infants with BW fulfilling current UK screening guidelines } \\
\hline GA $31+6$ weeks or less OR BW less than $1501 \mathrm{~g}$ & 8767 & 503 & 368 & 9638 & & & & \\
\hline \multicolumn{9}{|l|}{ Number of infants to be screened if guidelines tightened } \\
\hline GA $31+6$ weeks or less OR BW less than $1251 \mathrm{~g}$ & 7783 & 457 & 327 & 8567 & 11.2 & 9.1 & 11.1 & 11.1 \\
\hline GA $30+6$ weeks or less OR BW less than $1501 \mathrm{~g}$ & 7683 & 439 & 306 & 8428 & 12.4 & 12.7 & 16.8 & 12.6 \\
\hline GA $30+6$ weeks or less OR BW less than $1251 \mathrm{~g}$ & 6243 & 360 & 245 & 6848 & 28.8 & 28.4 & 33.4 & 28.9 \\
\hline GA $31+6$ & 7474 & 439 & 311 & 8224 & 14.7 & 12.7 & 15.4 & 14.7 \\
\hline GA $30+6$ & 5672 & 333 & 212 & 6217 & 35.3 & 33.8 & 42.4 & 35.5 \\
\hline
\end{tabular}

screening. The data covers the same time period as the national treatment study.

The first option would reduce the number of infants screened by 1071 babies or $11.1 \%$, the second by $12.6 \%$ (1210 babies), the third by $28.9 \%$ (2790 babies), the fourth by $14.7 \%$ (1414 babies), and the fifth by $35.5 \%$ (3421 babies) (Table 1). Options 1, 2, and 4 would have included all infants requiring treatment in the national treatment study cohort. Option 3 would have missed one infant who required treatment (GA 31+0 weeks, BW $1400 \mathrm{~g}$ ) and narrowly included another (GA 30+6 weeks, BW $1480 \mathrm{~g}$ ), and option 5 would have missed the baby of $31+0$ weeks GA.

A previous report from the NDAU has cautioned that reducing the screening criteria to $<31$ weeks GA or BW $<1251 \mathrm{~g}$ (scenario 3) would over a three-year period from 2009 to 2011 have missed eight babies requiring treatment. $^{10}$

Based on these figures, it appears safe to tighten the UK ROP screening guidelines to include infants with a GA of $31+6$ weeks or less or BW less than $1251 \mathrm{~g}$ (scenario 1), or those with GA of $30+6$ weeks or BW less than $1501 \mathrm{~g}$ (scenario 2). It would not be safe to lower both GA and BW cutoffs (scenario 3). Alternatively, an age only inclusion criteria could be used which, based on our data, would need to be $31+6$ or less (scenario 4 ). The risk of only using GA as an inclusion criteria is that occasionally infants born at over 32 weeks GA may have a very low BW due to growth restriction. However, the effect of growth restriction as an independent risk factor for ROP is unknown. Although uncertain GA was an important consideration in an earlier age, in well-developed healthcare systems with good obstetric care and ultrasound dating, this is now an unusual event.

Tightening the guidelines would spare $11-14.7 \%$ of infants the distress of repeated screening assessments, and reduce the economic burden of screening to the NHS.

We suggest that further prospective research analysing screening and treatment data from both ophthalmology and neonatal sources might allow further refinement in guidelines.

\section{Conflict of interest}

The authors declare no conflict of interest.

\section{Acknowledgements}

We thank Daniel Gray and Eugene Statnikov from the Neonatal Data Analysis Unit for supplying data from the National Neonatal Research Database. We thank the Moorfields Special Trustees (Grant ST 1401 D) and the Birmingham Eye Foundation for their generous funding for the British Ophthalmic Surveillance Unit study of retinopathy of prematurity requiring treatment, which has informed this work. The research was supported by the National Institute for Health Research (NIHR) Biomedical Research Centre based at Moorfields Eye Hospital NHS Foundation Trust and UCL Institute of Ophthalmology. The views expressed are those of the authors and not necessarily those of the NHS, the NIHR, or the Department of Health. Supported in part by the National Institute for Health Research Biomedical Research Centre at Moorfields Eye Hospital NHS Foundation Trust and UCL Institute of Ophthalmology.

\section{Members of the UK ROP Special Interest Group:}

Abbott, Joseph; Aclimandos, Wagih; Adams, Gill; Al-Khaier, Ayman; Allen, Louise; Arashvan, Kayvan; Ashworth, Jane; Barampouti, Faye; Barnes, Jonathan; Barrett, Victoria; Barry, John Sebastian; Bates, Adam; Berk, Tulin; Biswas, Susmito; Blaikie, Andrew; Brennan, Rosie; Bunting, Howard; Butcher, Jeremy; Butler, Lucilla; Chan-Ling, Tailoi; Chan, Jonathan; Child, Christopher; Choi, Jessy; Clark, David; Clifford, Luke; Dabbagh, Ahmad; Dahlmann-Noor, Annegret; Dawidek, Gervase; Dhir, Luna; Drake, Karen; Edwards, Richard; 
Esakowitz, Leonard; Escardo-Paton, Julia;

Evans, Anthony; Fleck, Brian; Geh, Vernon; George, Nick; Gnanaray, Lawrence; Goyal, Raina; Haigh, Paul; Hancox, Joanne; Haynes, Richard; Heath, Dominic; Henderson, Robert; Hillier, Roxane; Hingorani, Melanie; Jain, Saurabh; Jain, Sunila; Jones, David; Kafil-Hussain, Namir; Kelly, Simon; Kenawy, Nihal; Kipioti, Tina; Kulkarni, Archana; Lavy, Tim; Laws, David; Lawson, Joanna; Leitch, Jane; Ling, Roland; Long, Vernon; Macrae, Mary; Mahmood, Usman; Markham, Richard; Marr, Jane; May, Kristina; McLoone, Eibhlin; Moosa, Murad; Morton, Claire; Mount, Ali; Muen, Wisam; Mulvihill, Alan; Munshi, Vineeta; Muqit, Mahi; Murray, Robert; Nair, Ranjit; Newman, William; O'Colmain, Una; Patel, Chetan; Patel, Himanshu; Pedraza, Luis Amaya; Pilling, Rachel; Puvanachandra, Narman; Quinn, Anthony; Rathod, Dinesh; Reddy, Aravind; Reddy, Ashwin; Rowlands, Alison; Scotcher, Stephen; Scott, Christopher; Sekhri, Rajnish; Shafiq, Ayad; Sleep, Tamsin; Tambe, Katya; Tandon, Anamika; Tappin, Alison; Taylor, Robert; Theodoro, Maria; Thomas, Shery; Thompson, Graham; Tiffin, Peter; Ullah, Muhammed Aman; Watts, Patrick; West, Stephanie; Whyte, Iain; Wickham, Louisa; Williams, Cathy; Wong, Chien; Wren, Siobhan; Zakir, Rahila

\section{References}

1 Laws DE, Morton C, Weindling M, Clark D. Systemic effects of screening for retinopathy of prematurity. $\mathrm{Br} J$ Ophthalmol 1996; 80(5): 425-428.

2 Mukherjee AN, Watts P, Al-Madfai H, Manoj B, Roberts D. Impact of retinopathy of prematurity screening examination on cardiorespiratory indices: a comparison of indirect ophthalmoscopy and retcam imaging. Ophthalmology 2006; 113(9): 1547-1552.

3 Mehta M, Adams G, Bunce C, Xing W, Hill M. Pilot study of the systemic effects of three different screening methods used for retinopathy of prematurity. Early Hum Dev 2005; 81(4): 355-360.

4 Wiggins RE Jr, Gold RS, Menke AM. Twenty-five years of professional liability in pediatric ophthalmology and strabismus: the OMIC experience. J AAPOS 2015; 19(6): 535-540.
5 Fierson WM, American Academy of Pediatrics Section on O, American Academy of $\mathrm{O}$, American Association for Pediatric O, Strabismus, American Association of Certified O. Screening examination of premature infants for retinopathy of prematurity. Pediatrics 2013; 131(1): 189-195.

6 Jefferies AL. Canadian Paediatric Society F, Newborn C. Retinopathy of prematurity: An update on screening and management. Paediatr Child Health 2016; 21(2): 101-108.

7 Holmstrom G, Hellstrom A, Jakobsson P, Lundgren P, Tornqvist K, Wallin A. Evaluation of new guidelines for ROP screening in Sweden using SWEDROP - a national quality register. Acta Ophthalmol 2015; 93(3): 265-268.

8 Wilkinson AR, Haines L, Head K, Fielder AR. UK retinopathy of prematurity guideline. Early Hum Dev 2008; 84(2): 71-74.

9 Adams GG, Bunce C, Xing W, Butler L, Long V, Reddy A et al. Treatment trends for retinopathy of prematurity in the UK: active surveillance study of infants at risk. BMJ Open 2017; 7(3): e013366.

10 Wong HS, Santhakumaran S, Statnikov Y, Gray D, Watkinson M, Modi N et al. Retinopathy of prematurity in English neonatal units: a national population-based analysis using NHS operational data. Arch Dis Childhood Fetal Neonatal Edn 2014; 99(3): F196-F202.

GGW Adams ${ }^{1}$, C Williams², N Modi ${ }^{3}$, W Xing ${ }^{4}$, C Bunce ${ }^{5}$, UK Retinopathy of Prematurity Special Interest Group ${ }^{6}$ and A Dahlmann-Noor ${ }^{1,4}$

${ }^{1}$ Paediatric and Strabismus Service, Moorfields Eye Hospital NHS Foundation Trust, London, UK

${ }^{2}$ School of Social and Community Medicine, University of Bristol, Oakfield Grove, Clifton, UK

${ }^{3}$ Section of Neonatal Medicine, Department of Medicine, Imperial College London, London, UK

${ }^{4}$ National Institute of Health Research Biomedical Research Centre for Ophthalmology, University College London Institute of Ophthalmology and Moorfields Eye Hospital, London, UK

${ }^{5}$ Department of Primary Care \& Public Health Sciences, King's College London, London, UK

Correspondence: A Dahlmann-Noor, National Institute of Health Research Biomedical Research Centre for Ophthalmology, University College London Institute of Ophthalmology and Moorfields Eye Hospital, 162 City Road, London EC1V 2PD, UK.

E-mail: annegret.dahlmann-noor@moorfields.nhs.uk ${ }^{6}$ Members of the UK Retinopathy of Prematurity Special Interest Group are listed before the references. 